\title{
Stereospecific Interaction between Immune Cells and Chiral Surfaces
}

\section{Supportion Online Information}

\author{
Taolei Sun, ${ }^{*}$ Dong Han, Kristina Rhemann, Lifeng Chi, ${ }^{*}$ Harald Fuchs \\ Physicalisches Institut, Muenster University, D-48149 Muenster, Germany; National Center for Nanoscience \\ and Technology, Beijing 100080, P. R. China; Center for Nanoscience and Technology (CeNTech), D-48149 \\ Muenster, Germany \\ sunt@uni-muenster.de or Chi@uni-muenster.de
}

\section{Experimental details}

Preparation of the chiral surfaces: Gold was sputtered on smooth silicon wafers or the nanostructured PDMS substrates that fabricated by the imprinting technique using a preformed Ni template, with a thickness of about $15 \mathrm{~nm}$. Then they were put into the ethanol solutions of D-NIBC or L-NIBC (Fluka, $5 \mathrm{mg} / 10 \mathrm{~mL}$ ) under normal temperature. After immersion for about 12 hours, they were taken out and washed by ethanol excessively, and then dried by flowing nitrogen gas. The samples were immersed in the $75 \%$ medical ethanol solutions for several hours and then washed by PBS buffer solution before the cell experiments.

\section{Macrophage adhesion experiment:}

The human promyelocytic leukemia cellline HL-60, obtained from ATCC (Manassas; USA) was used in macrophage adhesion experiments. ${ }^{1}$ The differentiation to macrophage phenotype was induced by incubation in the presence of $50 \mathrm{ng} / \mathrm{mL}$ 12-tetradeconyl-phorbol 13-acetate (TPA) overnight. Differentiation was controlled microscopically.

After 24 hours of incubation at $37^{\circ} \mathrm{C}$ in $5 \% \mathrm{CO}_{2}$, and TPA the samples were washed twice with warm $\left(37^{\circ} \mathrm{C}\right)$ Celsuis PBS. After that, the cells were fixed with $1 \%$ Glutaraldehyd/PBS solution for 30 minutes, and then washed in warm PBS and A. dest for about 5 minutes, respectively. The cells were dehydrated in an ascending ethanol series of $50 \%, 70 \%, 80 \%, 95 \%$ ethanol, and absolute ethanol for 5 minutes, respectively and successively. Then the samples were preserved in ethanol for SEM observation.

\section{Neutrophil adhesion and activation experiments:}

Vein blood of a healthy human volunteer was collected via venipuncture and placed immediately into an anticoagulant tube (VACUTAINER Cat. No. 367946, BD). Then the whole blood was layered upon leucocyte separation medium (Ficoll, density $1.077 \mathrm{~g} / \mathrm{ml}$ ). Neutrophils were isolated by sedimentation of erythrocytes. Erythrocytes aggregated at the interface and settled at room temperature without centrifugation. After 40 min, the upper supernatant leukocyte-rich plasma was withdrawn, avoiding contact with plasma near the separation medium interface. To avoid factitious activation of neutrophil cells, the process did not include lysis, centrifugation, or washing. 
The supernatant leukocyte-rich plasma was suspended in Dulbecco's phosphate buffered saline (PBS) to a final concentration of about $1 \times 10^{9} / \mathrm{L}$. The neutrophils were incubated with the materals for $30 \mathrm{~min}$ at $37^{\circ} \mathrm{C}$.

Surface expression of CD11b and CD62L on neutrophils was assessed by direct immunofluorescence using mouse anti-human fluorochromecoupled monoclonal antibodies (CD11b-PE and CD62L-FITC). The cells were incubated with saturating amounts of antibodies for $30 \mathrm{~min}$ at $4 \mathrm{C}$ in the dark. After incubation, the cells were washed twice with BPS, and then analyzed by dual-fluorescence flow cytometry at laser excitation of $488 \mathrm{~nm}$.

\section{SEM observation:}

The cells were further dehydrated by supercritical $\mathrm{CO}_{2}$ drying process, and were imaged by the Field Emission SEM apparatus (JSM 6700F, Japan; and LEO VP 1530, Germany) after Ag sputtering.

\section{Immunofluorescene Results for Neutrophil Activation.}

(F1)[Ungated] 2CD62lfCD11bp.LMD : PMT1 Lin/FS Lin

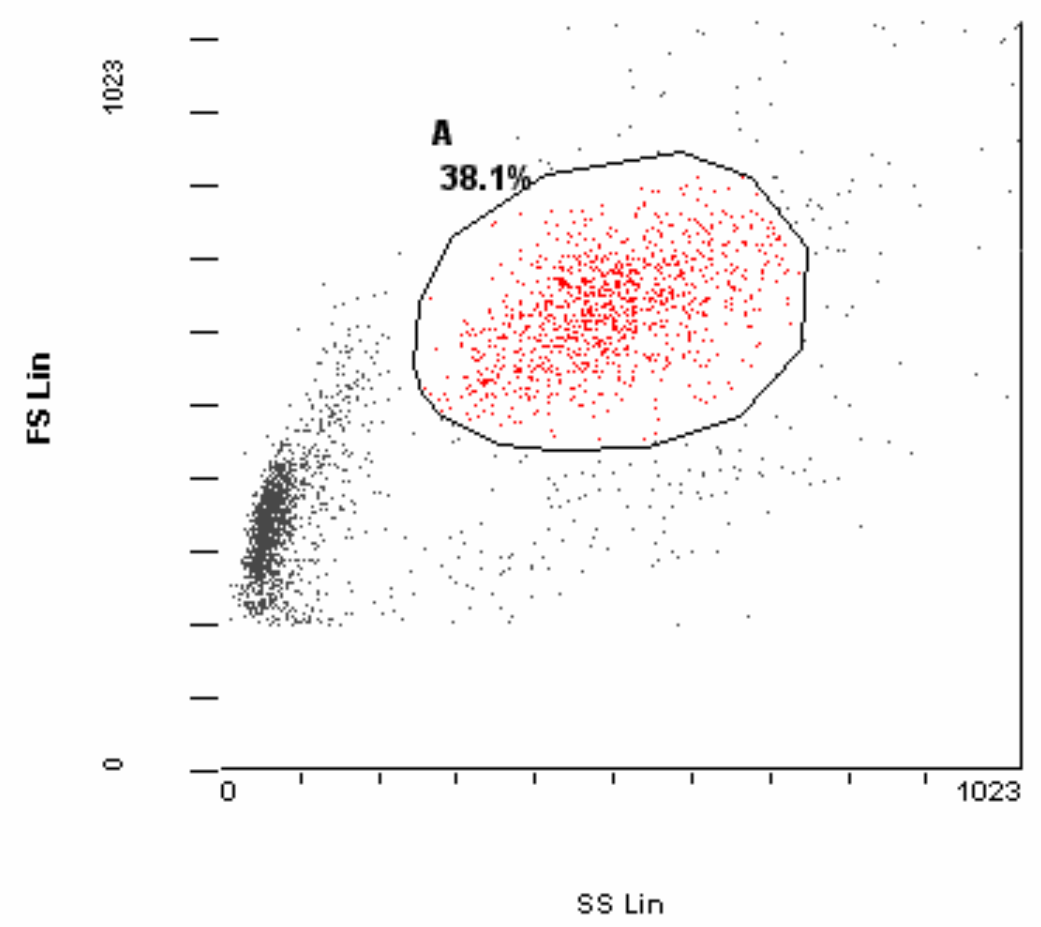

Figure S1. Gate selection for immunofluorescene experiments. 

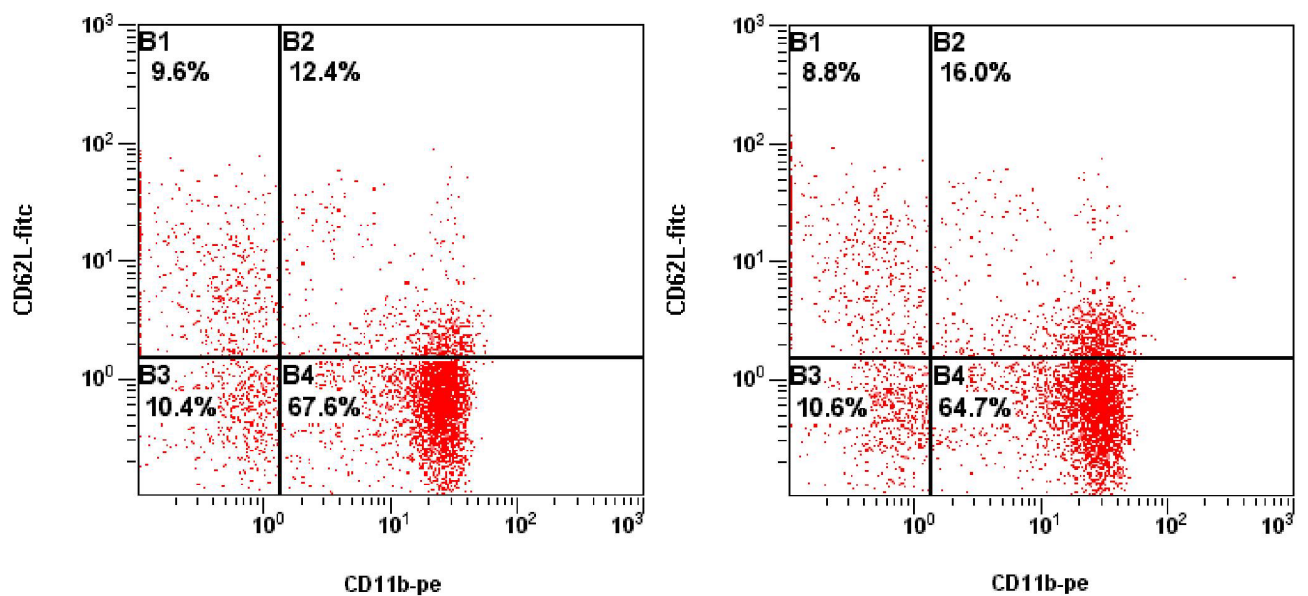

(a)
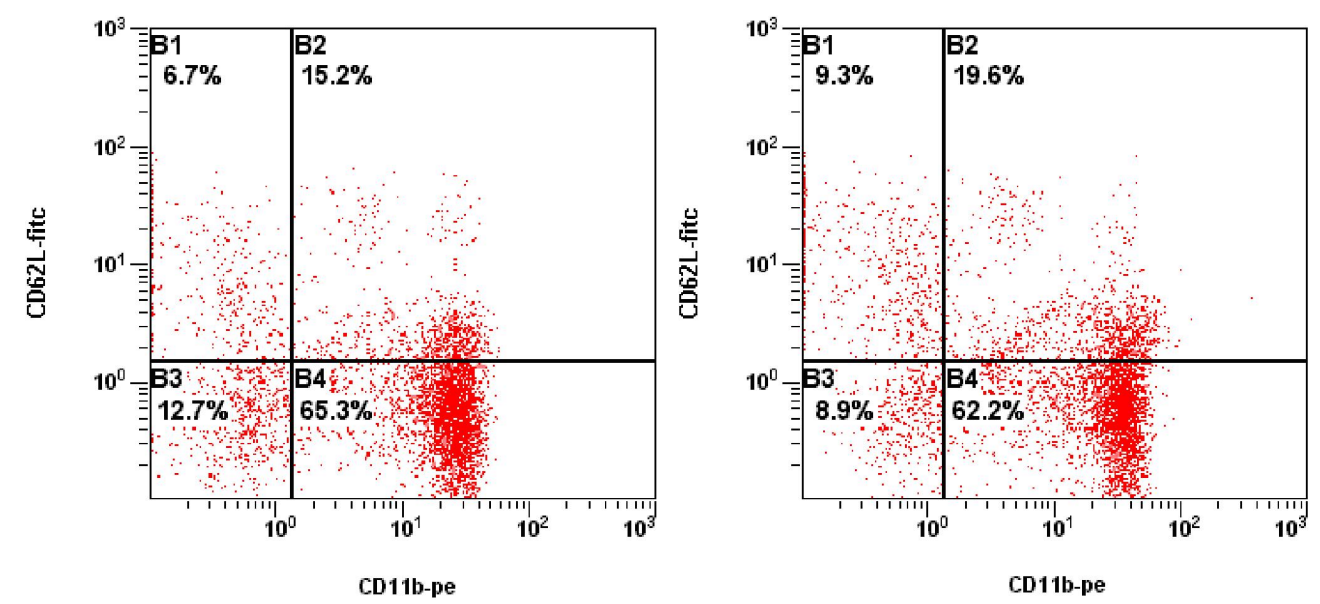

(b)

(c)
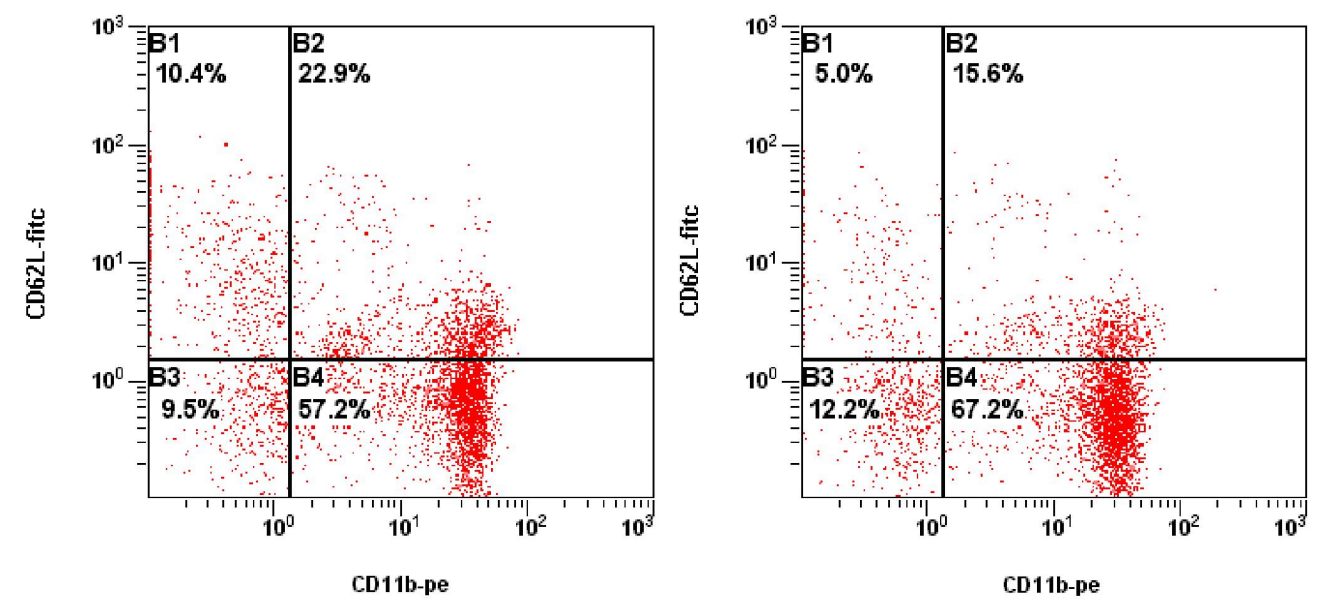

(e)

Figure S2. Immunofluorescene results for neutrophil activation. (a) D-NIBC modification on nanostructured substrate; (b) L-NIBC modification on nanostructured substrate; (c) Bare nanostructured substrate without NIBC modification; (d) D-NIBC modification on smooth substrate; (e) L-NIBC modification on smooth substrate; (f) Bare smooth substrate without NIBC modification. B2: $\mathrm{CD}_{11 \mathrm{~b}}{ } / \mathrm{CD} 62 \mathrm{~L}^{+}$events. Collected cell number for each analysis: 10,000 events. 


\section{References:}

1. Huberman, E; Callaham, M. F. Proc. Natl. Acad. Sci. USA 1979, 76, 1293-1297. 